\title{
Comparing Three Different Algorithms to Estimate Parameters of new Generated Marshal - Olkin Uniform Distribution
}

Prof. Dhwyia S. Hassan1/ Zainab F. Hamza2

1\&2Department of Business Information Technology,

College of Business Administration of Informatics,

University of Information Technology \&Communications, Iraq

\section{Abstract-}

This paper deals with constructing a new generated Marshal Olkin Uniform family distribution which include finding the probability density function $\left(p . d . f_{\text {), cumulative distribution }}\right.$ function $(C . D . F$, this new compound $p . d . f$ is necessary to represent the time to failure data of complex system, so we insist to extend Marshal - Olkin distribution to another family called Marshal - Olkin Weibull, we deriving the $(p . d . f$ ), ( $C . D . F$ ), and reliability function, then applying simulation procedure taking different sample size $n=20,40,80,100)$ and different set of initial values of $(\alpha, \theta$ ), each experiment repeated $(R=1000$ ), all results explained in tables.

Index Terms-MOEU(Marshall-Olkin extended uniform, MLE,Maximum likelihood estimator,MOM,Moment estimators, REM,Regresion estimators

\section{INTRODUCTION}

The p.d.f of Marshal - Olkin extended uniform (MOEU) is defined by;

$$
f\left(x_{*}^{*}, \alpha, \theta\right)=\frac{\alpha \theta}{[\alpha \theta+(1-\alpha) x]^{2}} \quad 0<x<\theta, \alpha>0
$$

Where; $(\alpha)$ is the shape parameter and $(\theta)$ is the scale parameter, while the corresponding cumulative distribution function is; (16)

$$
F(x ; \alpha, \theta)=\frac{x}{[\alpha \theta+(1-\alpha) x]} \quad 0<x<\theta, \quad \alpha>0
$$

The reliability function of (MOEU) is;

$$
R\left(x_{i}, \alpha, \theta\right)=\frac{\alpha(\theta-x)}{[\alpha \theta+(1-\alpha) x]} \quad 0<x<\theta, \alpha>0
$$

For the $p \cdot d . f$ in equation (1) we observe that the shape of this $p . d_{0} f, f\left(x_{*}, \alpha_{v}, \theta\right)$ depend on parameter $(\alpha)$, when $[\alpha \in(0,1)]$, the $p . d . f$ is decreasing function $(0, \theta)$ with;

$$
f(0 ; \alpha, \theta)=\frac{1}{\alpha \theta} \text { and } f(\theta ; \alpha, \theta)=\frac{\alpha}{\theta}
$$

Hayder A. Ameer3

MSc Operation Research
But when $(\alpha>1)$, the p.d.f is an increasing function on $(0, \theta)$ with (4). Also we can find hazard rate of the random variable $(x)$, which has p.d.f in (1);

(5)

$$
h\left(x_{;}^{*}, \alpha, \theta\right)=\frac{\theta}{[\alpha \theta+(1-\alpha) x](\theta-x)}
$$

The $r^{\text {th }}$ moment about origin of this distribution $[\operatorname{MOEU}(\alpha, \theta)]$ is;

$$
\begin{aligned}
& \mu_{r}^{g}=E\left(x^{r}\right) \int_{0}^{\theta} x^{r} f\left(x_{;}^{*} \alpha, \theta\right) d x \\
& =\frac{\alpha \theta}{(1-\alpha)^{\gamma+1}} \sum_{g=0}^{r} \frac{r !(-\alpha \theta)^{g}}{(r-s) ! s !(r-s-1)}\left[(\theta)^{r-s-1}-(\alpha \theta)^{\gamma-g-1}\right]
\end{aligned}
$$

According to this formula, the mean and variance of $r . v x$ with $[\operatorname{MOEU}(\alpha, \theta)]$ is;

$$
\mu_{1}^{t}=\frac{\alpha \theta}{(1-\alpha)^{2}}(\alpha-\log \alpha-1)
$$

$$
\mu_{2}^{s}=\frac{\alpha \theta^{2}}{(1-\alpha)^{4}}\left[(1-\alpha)^{2}-\alpha(\log \alpha)^{2}\right]
$$

Also we can show that the coefficient of variation is;

(9)

$$
c . v=\frac{\sqrt{\left[(1-\alpha)^{2}-\alpha(\log \alpha)^{2}\right]}}{\sqrt{\alpha}(\alpha-\log \alpha-1)} \quad \alpha>0
$$

Which depend on shape parameter $(\alpha)$, and the coefficient of Skewness is;

Where;

$$
S_{k}=\frac{\mu_{x}-\mu_{0}}{\sigma_{x}}
$$

We can prove that;

$$
\mu_{0}=x=\frac{\alpha \theta}{\alpha-1}
$$

$$
S_{k}=\frac{-\sqrt{\alpha} \ln \alpha}{\sqrt{\left[(1-\alpha)^{2}-\alpha(\ln \alpha)^{2}\right]}}
$$

\section{ESTIMATION OF THE PARAMETERS}

This section deals with introducing some methods to estimate two parameters $(\alpha, \theta)$, then applying simulation procedure to compare the results, these methods are;

II.1 MAXIMUM LIKELIHOOD METHOD

The estimators by this method obtained from maximizing; 


$$
\begin{gathered}
L(\alpha, \theta)=\frac{(\alpha \theta)^{n}}{\prod_{i=1}^{2}\left[\alpha \theta+(1-\alpha) x_{i}\right]^{2}} \\
\ln L=n L(\alpha, \theta)-2 \sum_{i=1}^{n} \ln \left[\alpha \theta+(1-\alpha) x_{i}\right] \\
\frac{\partial \ln L}{\partial \alpha}=\frac{n}{\alpha}-2 \sum_{i=1}^{n} \frac{\left(\theta-x_{i}\right)}{\left[\alpha \theta+(1-\alpha) x_{i}\right]}
\end{gathered}
$$

If we consider $\left[\hat{\theta}_{M L E}=x_{(m)}\right]$, then;

$\hat{\alpha}_{M L E}=\frac{n}{2}\left[\sum_{i=1}^{n-1} \frac{\left(x_{(n)}-x_{i}\right)}{\hat{a}_{M L E} x_{(n)}+\left(1-\hat{a}_{M L E}\right) x_{i}}\right]^{-1}$

Which is an implicit function can be solved numerically.

\section{II.2 MOMENT EstimatoR}

Let $x$ be $r, v \sim \operatorname{MOEU}(\alpha, \theta)$, then the moment estimators of $(\alpha, \theta)$ obtained from equating sample moment $\left[\mu_{y}=\frac{\sum_{i=1}^{n} x_{i}^{r}}{n}\right]$ with population moment $\left(\mu_{r}^{p}\right)$ defined in equation (6), we use coefficient of variation $\left(c_{*} v\right)$;

(14)

$$
\text { c.v }=\frac{\sqrt{\sqrt{E(x)}}}{E(x)}=\frac{\sqrt{\left[(1-\alpha)^{2}-\alpha(\ln \alpha)^{2}\right]}}{\sqrt{\alpha(-\ln \alpha-1+\alpha)}}
$$

Which is a function of shape parameter $(\alpha)$. Therefore equating $(c, v)$ with sample $(\boldsymbol{c} \cdot v)$;

$$
\frac{s}{\bar{s}}=\frac{\sqrt{\left[(1-\alpha)^{2}-\alpha(\ln \alpha)^{2}\right]}}{\sqrt{\alpha(-\ln \alpha-1+\alpha)}}
$$

This yield $\left(\hat{\alpha}_{\text {MOME }}\right)$ and then from $\left[E(x)=\mu_{1}^{g}\right]$, we can find $\left(\hat{\alpha}_{\text {MOME }}\right)$ :

$$
\hat{\alpha}_{M O M E}=\frac{\left.\overline{x_{(1-1}} \widehat{\tilde{\alpha}}_{M O M E}\right)^{2}\left(\widehat{\mathscr{\alpha}}_{M O M E}\right)^{-1}}{\left[\widehat{\tilde{a}}_{M O M E}-1-\ln \widehat{\alpha}_{M O M E}\right]}
$$

\section{II.3 Moment Estimator}

This method used to estimate $\left(\alpha_{s} \theta\right)$ for $\left[\operatorname{MOEU}\left(\alpha_{,} \theta\right)\right]$ is regression estimator which explained as follows; Let $\left(x_{1}, x_{2}, \ldots, x_{\eta}\right)$ be a random sample from $[\operatorname{MOEU}(\alpha, \theta)]$, defined in equation (1), then;

$$
\begin{aligned}
& \sqrt{F\left(x_{i}\right)}=\frac{\sqrt{\alpha \theta}}{\alpha \theta+(1-\alpha) x_{i}}=\frac{1}{\sqrt{\alpha \theta}+\frac{(1-\alpha)}{\sqrt{\alpha \theta} x_{i}}} \\
& \sqrt{\frac{1}{F\left(x_{i}\right)}}=\sqrt{\alpha \theta}+\frac{(1-\alpha)}{\sqrt{\alpha \theta}} x_{i}
\end{aligned}
$$

Let $y_{i} \Rightarrow \sqrt{\frac{1}{F\left(x_{i}\right)}} \quad \beta_{0}=\sqrt{\alpha \theta} \quad \beta_{1}=\frac{(1-\alpha)}{\sqrt{\alpha \theta}}$

$y_{i}=\beta_{0}+\beta_{1} x_{i}+u_{i}$ simple linear regression model, therefore;

(16)

$$
\hat{\alpha}_{R E}=1-\hat{\beta}_{0} \hat{\beta}_{1}
$$

And

$$
\hat{\theta}_{R E}=\frac{\beta_{0}^{2}}{1-\hat{\beta}_{0} \hat{\beta}_{1}}
$$

\begin{tabular}{|c|c|c|c|c|c|}
\hline $\mathbf{n}$ & Method & $\alpha$ & $\operatorname{MSE}(\alpha)$ & $\theta$ & $\operatorname{MSE}(\theta)$ \\
\hline \multirow{3}{*}{20} & MLE & 1.031278 & 0.113673 & 2.851806 & 0.039166 \\
\hline & Moment & 1.068291 & 0.276983 & 2.996657 & 0.001013 \\
\hline & Regression & 1.031082 & 0.11297 & 2.847869 & 0.040374 \\
\hline \multicolumn{2}{|r|}{ BEST } & \multicolumn{2}{|r|}{ MOM } & \multicolumn{2}{|r|}{ MOM } \\
\hline \multirow{3}{*}{40} & MLE & 1.00589 & 0.059886 & 2.937422 & 0.007572 \\
\hline & Moment & 1.019005 & 0.106815 & 2.999201 & 0.00036 \\
\hline & Regression & 1.005839 & 0.059516 & 2.933136 & 0.008074 \\
\hline \multicolumn{2}{|r|}{ BEST } & \multicolumn{2}{|r|}{ REG } & \multicolumn{2}{|r|}{ MOM } \\
\hline \multirow{3}{*}{80} & MLE & 1.068839 & 0.080575 & 2.949082 & 0.005078 \\
\hline & Moment & 1.107214 & 0.134356 & 3.000366 & 0.000264 \\
\hline & Regression & 1.068609 & 0.080022 & 2.944403 & 0.00561 \\
\hline \multicolumn{2}{|r|}{ BEST } & \multicolumn{2}{|r|}{ REG } & \multicolumn{2}{|r|}{ MOM } \\
\hline \multirow{3}{*}{100} & MLE & 0.992154 & 0.026968 & 2.971291 & 0.001815 \\
\hline & Moment & 0.984331 & 0.036687 & 2.999123 & 5.07E-05 \\
\hline & Regression & 0.992206 & 0.026804 & 2.966234 & 0.002137 \\
\hline & BEST & & REG & \multicolumn{2}{|r|}{$\mathrm{MOM}$} \\
\hline
\end{tabular}

\begin{tabular}{|c|c|c|c|c|c|}
\hline$n$ & Method & $\alpha$ & $\operatorname{MSE}(\alpha)$ & $\theta$ & $\operatorname{MSE}(\theta)$ \\
\hline \multirow{3}{*}{20} & MLE & 1.100314 & 0.238803 & 3.32512 & 0.059525 \\
\hline & Moment & 1.229697 & 0.510707 & 3.502232 & $2.10 \mathrm{E}-03$ \\
\hline & Regression & 1.099771 & 0.236671 & 3.318926 & 0.061781 \\
\hline \multicolumn{2}{|r|}{ BEST } & & REG & & MOM \\
\hline \multirow{3}{*}{40} & MLE & 1.066186 & 0.062155 & 3.443084 & 0.00695 \\
\hline & Moment & 1.084501 & 0.083461 & 3.499059 & $1.49 \mathrm{E}-04$ \\
\hline & $\begin{array}{l}\text { Regression } \\
\end{array}$ & 1.065977 & 0.061891 & 3.438127 & 0.007579 \\
\hline \multicolumn{2}{|r|}{ BEST } & & REG & & MOM \\
\hline \multirow{3}{*}{80} & MLE & 1.012072 & 0.034551 & 3.457482 & 0.003955 \\
\hline & Moment & 1.00564 & 0.069503 & 3.500705 & $1.36 \mathrm{E}-04$ \\
\hline & Regression & 1.012036 & 0.034326 & 3.451485 & 0.004553 \\
\hline \multicolumn{2}{|r|}{ BEST } & & REG & & MOM \\
\hline \multirow{3}{*}{100} & MLE & 0.996889 & 0.024841 & 3.465704 & 0.002261 \\
\hline & Moment & 1.005863 & 0.045934 & 3.500502 & $9.70 \mathrm{E}-05$ \\
\hline & Regression & 0.996893 & 0.024648 & 3.459205 & 0.002801 \\
\hline \multicolumn{2}{|r|}{ BEST } & & REG & & MOM \\
\hline
\end{tabular}

III. Application

The application has been done through simulation procedure to compare between the estimators, for the values of scale parameter $\left({ }^{\theta}\right)$ and shape parameter $\left({ }^{\alpha}\right)$ with $(n=20,40,80,100)$, the comparison for scale parameter and shape parameter was done by MSE.

\begin{tabular}{|l|l|l|l|}
\hline$\theta$ & 3 & 3.5 & 4 \\
\hline$\alpha$ & 1 & 1.5 & 2 \\
\hline
\end{tabular}

Table (1): $\alpha=1 \quad \theta=3$

Table (2): $\alpha=1 \quad \theta=3.5$ 
Table (3): $\alpha=1 \quad \theta=4$ Prepare Your PAPER

\begin{tabular}{|c|c|c|c|c|c|}
\hline $\mathrm{n}$ & Method & $\alpha$ & $\operatorname{MSE}(\alpha)$ & $\theta$ & $\operatorname{MSE}(\theta)$ \\
\hline \multirow{3}{*}{20} & MLE & 1.042908 & 0.138847 & 3.811649 & 0.063222 \\
\hline & Moment & 1.156049 & 0.287677 & 4.007926 & $1.98 \mathrm{E}-03$ \\
\hline & Regression & 1.042368 & 0.137414 & 3.804282 & 0.065918 \\
\hline \multicolumn{2}{|r|}{ BEST } & \multicolumn{2}{|r|}{ REG } & \multicolumn{2}{|r|}{ MOM } \\
\hline \multirow{3}{*}{40} & MLE & 1.029217 & 0.078406 & 3.911941 & 0.014818 \\
\hline & Moment & 1.043457 & 0.159298 & 4.002348 & $5.13 \mathrm{E}-04$ \\
\hline & Regression & 1.029042 & 0.07787 & 3.904754 & 0.01623 \\
\hline \multicolumn{2}{|r|}{ BEST } & \multicolumn{2}{|r|}{ REG } & \multicolumn{2}{|r|}{ MOM } \\
\hline \multirow{3}{*}{80} & MLE & 1.034246 & 0.034645 & 3.958564 & 0.004069 \\
\hline & Moment & 1.054223 & 0.060447 & 3.999109 & $1.51 \mathrm{E}-04$ \\
\hline & Regression & 1.034102 & 0.034334 & 3.950485 & 0.004775 \\
\hline \multicolumn{2}{|r|}{ BEST } & \multicolumn{2}{|r|}{ REG } & \multicolumn{2}{|r|}{ MOM } \\
\hline \multirow{3}{*}{100} & MLE & 1.029207 & 0.041988 & 3.969557 & 0.001767 \\
\hline & Moment & 1.006764 & 0.050583 & 3.999158 & $9.19 \mathrm{E}-05$ \\
\hline & Regression & 1.029003 & 0.041595 & 3.961514 & 0.002371 \\
\hline \multicolumn{2}{|r|}{ BEST } & \multicolumn{2}{|r|}{ REG } & \multicolumn{2}{|r|}{ MOM } \\
\hline
\end{tabular}

Table (4): $\alpha=1.5 \quad \theta=3$

\begin{tabular}{|c|c|c|c|c|c|}
\hline $\mathbf{n}$ & Method & $\alpha$ & $\operatorname{MSE}(\alpha)$ & $\theta$ & $\operatorname{MSE}(\theta)$ \\
\hline \multirow{3}{*}{20} & MLE & 1.603319 & 0.470726 & 2.898675 & 0.020577 \\
\hline & Moment & 1.677129 & 1.051682 & 3.000486 & $1.98 \mathrm{E}-03$ \\
\hline & Regression & 1.600803 & 0.465825 & 2.895817 & 0.021229 \\
\hline \multicolumn{2}{|r|}{ BEST } & \multicolumn{2}{|r|}{ REG } & \multicolumn{2}{|r|}{ MOM } \\
\hline \multirow{3}{*}{40} & MLE & 1.650908 & 0.240887 & 2.946342 & 0.005478 \\
\hline & Moment & 1.768623 & 0.535315 & 3.008162 & $6.91 \mathrm{E}-04$ \\
\hline & Regression & 1.648319 & 0.238308 & 2.943197 & 0.005813 \\
\hline \multicolumn{2}{|r|}{ BEST } & \multicolumn{2}{|r|}{ REG } & \multicolumn{2}{|r|}{ MOM } \\
\hline \multirow{3}{*}{80} & MLE & 1.519302 & 0.10133 & 2.972898 & 0.001614 \\
\hline & Moment & 1.544359 & 0.182681 & 3.001927 & $3.03 \mathrm{E}-04$ \\
\hline & Regression & 1.51761 & 0.100462 & 2.970086 & 0.001766 \\
\hline \multicolumn{2}{|r|}{ BEST } & \multicolumn{2}{|r|}{ REG } & \multicolumn{2}{|r|}{ MOM } \\
\hline \multirow{3}{*}{100} & MLE & 1.510354 & 0.064359 & 2.981114 & 0.00074 \\
\hline & Moment & 1.546363 & 0.168409 & 3.002502 & $2.96 \mathrm{E}-04$ \\
\hline & Regression & 1.50853 & 0.063907 & 2.977822 & 0.000893 \\
\hline \multicolumn{2}{|r|}{ BEST } & \multicolumn{2}{|r|}{ REG } & \multicolumn{2}{|r|}{ MOM } \\
\hline
\end{tabular}

Table (5): $\alpha=1.5 \theta=3.5$

\begin{tabular}{|c|c|c|c|c|c|}
\hline $\mathbf{n}$ & Method & $\alpha$ & $\operatorname{MSE}(\alpha)$ & $\theta$ & $\operatorname{MSE}(\theta$ \\
\hline \multirow{3}{*}{$\begin{array}{l}2 \\
0\end{array}$} & MLE & 1.662229 & 0.482339 & 3.395761 & 0.01763 \\
\hline & Moment & 1.75725 & 0.731479 & 3.500527 & $\begin{array}{c}2.40 \mathrm{E}- \\
03\end{array}$ \\
\hline & Regression & 1.659424 & 0.477065 & 3.391316 & $\begin{array}{c}0.01869 \\
3\end{array}$ \\
\hline \multicolumn{2}{|r|}{ BEST } & & REG & & MOM \\
\hline \multirow[t]{3}{*}{40} & MLE & 1.50306 & 0.184362 & 3.436737 & $\begin{array}{c}0.00713 \\
6\end{array}$ \\
\hline & Moment & 1.556405 & 0.355055 & 3.502946 & $\begin{array}{c}8.79 \mathrm{E}- \\
04\end{array}$ \\
\hline & Regression & 1.500762 & 0.182108 & 3.43237 & $\begin{array}{c}0.00769 \\
6\end{array}$ \\
\hline \multicolumn{2}{|r|}{ BEST } & & REG & & MOM \\
\hline \multirow[t]{3}{*}{80} & MLE & 1.531236 & 0.065936 & 3.47274 & $\begin{array}{c}0.00164 \\
3\end{array}$ \\
\hline & Moment & 1.565229 & 0.134749 & 3.501977 & $\begin{array}{c}.93 \mathrm{E}- \\
04\end{array}$ \\
\hline & Regression & 1.529203 & 0.065282 & 3.468705 & $\begin{array}{c}0.00191 \\
3\end{array}$ \\
\hline \multicolumn{2}{|r|}{ BEST } & & REG & & MOM \\
\hline \multirow[t]{3}{*}{100} & MLE & 1.50272 & 0.050678 & 3.476985 & $\begin{array}{c}0.00094 \\
1\end{array}$ \\
\hline & Moment & 1.533148 & 0.159062 & 3.502192 & $\begin{array}{c}3.75 \mathrm{E}- \\
04\end{array}$ \\
\hline & Regression & 1.500694 & 0.05031 & 3.472647 & $\begin{array}{c}0.00115 \\
8\end{array}$ \\
\hline
\end{tabular}

Table (6): $\alpha=1.5 \quad \theta=4$

\begin{tabular}{|c|c|c|c|c|c|}
\hline $\mathbf{n}$ & Method & $\alpha$ & $\operatorname{MSE}(\alpha)$ & $\theta$ & $\operatorname{MSE}(\theta)$ \\
\hline \multirow[t]{3}{*}{20} & MLE & 1.602384 & 0.368624 & 3.869891 & $\begin{array}{c}0.03230 \\
5\end{array}$ \\
\hline & Moment & 1.825823 & 1.436017 & 4.015709 & $\begin{array}{c}3.21 \mathrm{E}- \\
03\end{array}$ \\
\hline & Regression & 1.598837 & 0.362828 & 3.863818 & $\begin{array}{c}0.03407 \\
8 \\
\end{array}$ \\
\hline \multicolumn{2}{|r|}{ BEST } & \multicolumn{2}{|r|}{ REG } & \multicolumn{2}{|r|}{ MOM } \\
\hline \multirow[t]{3}{*}{40} & MLE & 1.551459 & 0.185092 & 3.93784 & $\begin{array}{c}0.00737 \\
7\end{array}$ \\
\hline & Moment & 1.605304 & 0.440727 & 4.002972 & $\begin{array}{c}1.27 \mathrm{E}- \\
03\end{array}$ \\
\hline & Regression & 1.548679 & 0.183124 & 3.932044 & 0.00812 \\
\hline \multicolumn{2}{|r|}{ BEST } & \multicolumn{2}{|r|}{ REG } & \multicolumn{2}{|r|}{ MOM } \\
\hline \multirow[t]{3}{*}{80} & MLE & 1.549335 & 0.101966 & 3.970651 & $\begin{array}{c}0.00163 \\
6\end{array}$ \\
\hline & Moment & 1.619259 & 0.251243 & 4.005788 & $\begin{array}{c}7.38 \mathrm{E}- \\
04 \\
\end{array}$ \\
\hline & Regression & 1.546586 & 0.100513 & 3.964991 & $\begin{array}{c}0.00204 \\
2\end{array}$ \\
\hline \multicolumn{2}{|r|}{ BEST } & \multicolumn{2}{|r|}{ REG } & \multicolumn{2}{|r|}{ MOM } \\
\hline \multirow{3}{*}{$\begin{array}{c}10 \\
0\end{array}$} & MLE & 1.511176 & 0.076053 & 3.971001 & $\begin{array}{c}0.00180 \\
2\end{array}$ \\
\hline & Moment & 1.52876 & 0.151724 & 4.002049 & $\begin{array}{c}3.62 \mathrm{E}- \\
04\end{array}$ \\
\hline & Regression & 1.508754 & 0.075092 & 3.965391 & $\begin{array}{c}0.00217 \\
1\end{array}$ \\
\hline & BEST & \multicolumn{2}{|r|}{ REG } & \multicolumn{2}{|r|}{ MOM } \\
\hline
\end{tabular}

Table (7): $\quad \alpha=2 \quad \theta=3$

\begin{tabular}{|c|c|c|c|c|c|}
\hline $\mathbf{n}$ & Method & $\alpha$ & $\operatorname{MSE}(\alpha)$ & $\theta$ & $\operatorname{MSE}(\theta)$ \\
\hline \multirow{3}{*}{20} & MLE & 2.203633 & 0.877015 & 2.936145 & 0.008075 \\
\hline & Moment & 2.510654 & 2.760199 & 3.012835 & $2.48 \mathrm{E}-03$ \\
\hline & Regression & 2.199787 & 0.869804 & 2.93423 & 0.00837 \\
\hline \multicolumn{2}{|r|}{ BEST } & & REG & & MOM \\
\hline \multirow{3}{*}{40} & MLE & 2.141178 & 0.345645 & 2.9709 & 0.00167 \\
\hline & Moment & 2.313537 & 1.099686 & 3.006987 & $1.55 \mathrm{E}-03$ \\
\hline & Regression & 2.136453 & 0.341346 & 2.968616 & 0.001801 \\
\hline \multicolumn{2}{|r|}{ BEST } & & REG & & MOM \\
\hline \multirow{3}{*}{80} & MLE & 2.034213 & 0.137878 & 2.982707 & 0.000516 \\
\hline & Moment & 2.139557 & 0.554344 & 3.004001 & $9.57 \mathrm{E}-04$ \\
\hline & Regression & 2.030249 & 0.136308 & 2.980478 & 0.000607 \\
\hline \multicolumn{2}{|r|}{$\begin{array}{l}\text { BEST } \\
\end{array}$} & & REG & & MOM \\
\hline \multirow{3}{*}{100} & MLE & 1.982068 & 0.133995 & 2.985069 & 0.000551 \\
\hline & Moment & 1.969524 & 0.263091 & 2.99837 & $6.01 \mathrm{E}-04$ \\
\hline & Regression & 1.978385 & 0.132882 & 2.98279 & 0.000631 \\
\hline & BEST & & REG & & MOM \\
\hline
\end{tabular}

Table (8): $\alpha=2 \quad \theta=4$

\begin{tabular}{|c|c|c|c|c|c|}
\hline $\mathbf{n}$ & Method & $\alpha$ & $\operatorname{MSE}(\alpha)$ & $\boldsymbol{\theta}$ & $\operatorname{MSE}(\theta)$ \\
\hline \multirow{3}{*}{20} & MLE & 2.213872 & 0.653475 & 3.898859 & 0.01965 \\
\hline & Moment & 2.798112 & 6.377826 & 4.022145 & $7.83 \mathrm{E}-03$ \\
\hline & Regression & 2.207895 & 0.644301 & 3.895201 & 0.020476 \\
\hline \multicolumn{2}{|r|}{$\begin{array}{l}\text { BEST } \\
\end{array}$} & & REG & & MOM \\
\hline \multirow{3}{*}{40} & MLE & 2.109576 & 0.409909 & 3.942162 & 0.007259 \\
\hline & Moment & 2.266288 & 0.997309 & 4.010185 & $3.08 \mathrm{E}-03$ \\
\hline & Regression & 2.103151 & 0.401116 & 3.938412 & 0.007763 \\
\hline \multicolumn{2}{|r|}{ BEST } & & REG & & MOM \\
\hline \multirow{3}{*}{80} & MLE & 2.038608 & 0.150793 & 3.977039 & 0.001124 \\
\hline & Moment & 2.114785 & 0.365654 & 4.004357 & $1.43 \mathrm{E}-03$ \\
\hline & Regression & 2.033227 & 0.148042 & 3.973238 & 0.001312 \\
\hline \multicolumn{2}{|r|}{ BEST } & & REG & & MOM \\
\hline \multirow{3}{*}{100} & MLE & 2.081594 & 0.142793 & 3.984962 & 0.00041 \\
\hline & Moment & 2.095828 & 0.26858 & 3.999847 & $9.30 \mathrm{E}-04$ \\
\hline & Regression & 2.076102 & 0.140141 & 3.981166 & 0.000536 \\
\hline & BEST & & REG & & MOM \\
\hline
\end{tabular}




\section{CONCLUSION}

From table one the best estimator is $\alpha$ (where $\operatorname{MSE}(\alpha$ )$=0.026804)$ REG and the best estimator for $\theta$ is $\hat{\theta}_{\text {MOME }}$ ,since $\operatorname{MSE}(\theta)=0.002137$ also from table two , we find $\hat{\alpha}_{R E}$ is best and $\hat{\theta}_{M O M}$ is best, where $\operatorname{MSE}\left(\hat{\alpha}_{R E}\right)=0.024648$ $\operatorname{MSE}\left(\hat{\theta}_{\text {MOM }}\right)=0.002801$

Also from table three, we find best estimator is $\hat{\alpha}_{R E}$ and $\hat{\theta}_{\text {MOM }}$, similarly from table four we find best estimator for $\alpha$ is $\hat{\alpha}_{R E}$ and for $\theta$ is $\hat{\theta}_{M O M}$

\section{RECOMMENDATION}

Since this constructed model represent a new generated Marshal - Olkin uniform family, and it have two parameters $(\alpha)$ shape parameter , $\theta$ is scale parameter so we use simulation taking sample size $(n=20,40,80,100)$ and each experiment is Repeated 1000 times to estimate these two parameters, and the results are compared by statistical measure (Mean Square error) and the results is explained in tables .

\section{REFERENCES}

[1] [1] Al _ Athari, Faris, M. (2011), "Parameter Estimation for the double Pareto Distribution", Journal of mathematics and statistics, 7(4):289-294.

[2] [2] Alice, T., Jose, K.K., Marshall- Olkin Pareto Processes, Far East Journal of Theoretical Statistics, (2003), 9(2), 117132.

[3] [3] Arwa Y. Al-Saiari, Lamya A. Baharith \& Salwa A. Mousa, (2014)," Marshall-Olkin Extended Burr Type XII Distribution", International Journal of Statistics and Probability; Vol. 3, No. 1

[4] [4] Ashwini K. Srivastava, Vijay Kumar, (2011), " Software Reliability Data Analysis with Marshall-Olkin Extended Weibull Model using MCMC Method for Non-Informative Set of Priors", International Journal of Computer Applications (0975 - 8887), Volume 18-No.4.

[5] [5] Cordeiro, G. M., \& Lemonte, A. J. (2013). On the Marshall-Olkin Extended Weibull Distribution. Stat Papers, 54،333-353. http://dx.doi.org/10.1007/s00362-012-0431-8
[6] [6] Debasis Kundu, Arabin Kumar Dey, (2009), " Estimating the parameters of the Marshall-Olkin bivariate Weibull distribution by EM algorithm", Journal of Computational Statistics \& Data Analysis archive Volume 53 Issue 4, PP. 956-965.

[7] [7] Gui, W. (2013). A Marshall-Olkin Power Log-normal Distribution and Its Applications to Survival Data. International Journal of Statistics and Probability, 2(1), 63-72. http://dx.doi.org/10.5539/ijsp.v2n1p63

[8] [8] Gupta, A.K., Nadaarajah, S. (2004), " on the moments of the beta normal distribution", Commun. Stat. Theory and Methods, 33: 1-13.

[9] [9] Jose, K. and Krishna, E. (2011), "Marshal Olkin extended uniform distribution" Prob. Stat. Forum, Vol. 04, 78 - 88.

[10] [10] Jones, M. C. (2004), "Families of distributions arising from distributions of order statistics" Test 13; 1-43.

[11] [11] Manoel Santos-Neto, Marcelo Bourguignon, Luz M Zea, Abraão DC Nascimento and Gauss M Cordeiro, (2014), "The Marshall-Olkin extended Weibull family of distributions", journal of Statistical Distributions and Applications, Vol.1.

[12] [12] Marshal, A.W., Olkin, I., (1997) " A new method for adding a parameter to a family of distributions with applications to the exponential and Weibull families" Biometrica, 84,641- 652.

[13] [13] Qiang Guan, Yincai Tang, Ancha Xu, (2013), " Objective Bayesian analysis for bivariate Marshall-Olkin exponential distribution", Computational Statistics \& Data Analysis Volume 6.

[14] [14] Ristic, M. M., Jose, K. K., \& Ancy, J. A. (2007). Marshall Olkin gamma distribution and minification process. Stars: Stress and Anxiety Research Society, 11, 107-117.

[15] [15] Saleh S. Muhammed (2006), " A comparison Bayesian approach with another methods to estimate reliability function for Pareto distribution" Master of Science thesis, department of statistics, Baghdad University.

[16] [16] Salah H. Abid, Heba A. Hassan, (2015), "The MarshallOlkin Extended Uniform Stress-Strength Model", American Journal of Mathematics and Statistics, 5(1): 1-10.

[17] [17] Srivastava, A. K., Kumar, V., Hakkak, A. A., \& Khan, M. A. (2011). Estimation of Marshall-Olkin Extended Exponential distribution Parameter using Markov Chain Monte Carlo Method for Informative set of Priors. International Journal of Advances in Science and Technology, 2(4), 68-92. 